\title{
Killing of adherent oral microbes by a non-thermal atmospheric plasma jet

\begin{abstract}
Correspondence
Stefan Rupf

stefan.rupf@uks.eu
\end{abstract} \\ Received 19 June 2009 \\ Accepted 5 November 2009

\author{
Stefan Rupf, ${ }^{1}$ Antje Lehmann, ${ }^{2}$ Matthias Hannig, ${ }^{1}$ Barbara Schäfer, ${ }^{1}$ \\ Andreas Schubert, ${ }^{3}$ Uwe Feldmann $^{4}$ and Axel Schindler ${ }^{2}$ \\ ${ }^{1}$ Clinic of Operative Dentistry, Periodontology and Preventive Dentistry, Saarland University, \\ Homburg, Germany \\ ${ }^{2}$ Leibniz Institute for Surface Modification, Leipzig, Germany \\ ${ }^{3}$ Vascular Biology Group, Fraunhofer Institute, Leipzig, Germany \\ ${ }^{4}$ Institute for Medical Biometry, Epidemiology and Medical Informatics, Saarland University, \\ Homburg, Germany
}

\begin{abstract}
Atmospheric plasma jets are being intensively studied with respect to potential applications in medicine. The aim of this in vitro study was to test a microwave-powered non-thermal atmospheric plasma jet for its antimicrobial efficacy against adherent oral micro-organisms. Agar plates and dentin slices were inoculated with $6 \log _{10}$ c.f.u. $\mathrm{cm}^{-2}$ of Lactobacillus casei,

Streptococcus mutans and Candida albicans, with Escherichia coli as a control. Areas of $1 \mathrm{~cm}^{2}$ on the agar plates or the complete dentin slices were irradiated with a helium plasma jet for 0.3 , 0.6 or $0.9 \mathrm{~s} \mathrm{~mm}^{-2}$, respectively. The agar plates were incubated at $37{ }^{\circ} \mathrm{C}$, and dentin slices were vortexed in liquid media and suspensions were placed on agar plates. The killing efficacy of the plates, as well as by determination of the number of c.f.u. recovered from dentin slices. A microbealbicans and E. coli. The plasma-jet treatment reduced the c.f.u. by $3-4 \log _{10}$ intervals on the dentin slices in comparison to recovery rates from untreated controls. The microbe-killing effect was correlated with increasing irradiation times. Thus, non-thermal atmospheric plasma jets could be used for the disinfection of dental surfaces.
\end{abstract} plasma jet was assessed by counting the number of c.f.u. on the irradiated areas of the agar killing effect was found on the irradiated parts of the agar plates for L. casei, S. mutans, C.
}

\section{INTRODUCTION}

Plasma jets are ionized local gas flows, generated under normal pressure by means of microwaves, high frequency or pulsed direct current in so-called plasma-jet sources using noble gases. Reactive oxygen species are produced through an admixture of chemically active gases $\left(\mathrm{O}_{2}, \mathrm{~N}_{2}\right.$ and others), which are able to react with biological material or tissues (Kieft et al., 2005). Furthermore, UV irradiation, ions and electrons are emitted in the plasma discharges, which also interact with biological material or tissues (Laroussi \& Leipold, 2004; Vleugels et al., 2005). In medicine, plasmas are used to sterilize surgical instruments and consumables (Masaoka, 2007). Another important medical application is argon-plasma coagulation (Manner, 2008). Plasmas can also be used to give hydrophilic properties to surfaces (Duan et al., 2007).

Recently, the generation of local plasmas under atmospheric pressure with low temperatures of around $40{ }^{\circ} \mathrm{C}$

Abbreviations: $\mathrm{CHX}$, chlorhexidine; $\mathrm{Cl}$, confidence interval; SEM, scanning electron microscopy. has been achieved. At the same time much more compact plasma sources were developed making them more attractive for medical applications (Coulombe et al., 2006; Yu et al., 2006; Sladek et al., 2007). The antibacterial effect of cold plasmas has been shown for a variety of micro-organisms in numerous studies (Moisan et al., 2001; Laroussi, 2002; Becker et al., 2005; Laroussi \& Lu, 2005; Vleugels et al., 2005; Lee et al., 2006; Yu et al., 2006, 2007; Hong et al., 2009). However, some studies have shown that bacterial DNA is not completely destroyed by cold plasma (Venezia et al., 2008; Kim \& Kim, 2006).

Methods for the decontamination and conditioning of intraoral surfaces are of great interest in the field of dentistry. Cold plasmas are of particular interest, as heat damage of the dental pulp must be prevented. Only a few papers have been published dealing with the application of plasma jets for biofilm and plaque removal in dentistry. For instance, the removal of carious dentin has been suggested as an alternative to conventional drilling (Stoffels et al., 2002; Sladek et al., 2004). The in-vitro disinfection of Streptococcus mutans grown on agar plates (Goree et al., 
2006) and in biofilms has been demonstrated (Sladek et al., 2007). However, due to the presence of dentin tubules, the disinfection of dentin poses special challenges during caries therapy. It must be achieved either by invasive removal, by chemical disinfection or by application of ozone. No data on the effect of plasma jets on microbially contaminated dentin are available yet.

The aim of this in vitro study was to test a non-thermal atmospheric plasma jet for its antimicrobial efficacy against important oral micro-organisms grown on agar plates or adherent to dentin slices. The experiments were performed with the caries-associated bacteria Lactobacillus casei (Badet \& Thebaud, 2008) and S. mutans (Rupf et al., 2008), as well as Candida albicans (Klinke et al., 2009), which is frequently isolated from dentin caries lesions. Escherichia coli was included as a control in the study.

\section{METHODS}

Plasma jet. A non-thermal plasma source (microwave driven, 2.45 $\mathrm{GHz}$ ) (Fig. 1) developed at the Leibniz Institute of Surface Modification was mounted on a computer controlled three-axis motion system to ensure reproducible time, distance and scanning parameters. Irradiation was carried out at a distance of $1.5 \mathrm{~mm}$ between plasma-jet nozzle and sample surface. The pulse width of the microwave was $5 \mu$ s at $300 \mathrm{~W}$, and the process gas flow was $\mathrm{He} / \mathrm{O}_{2} / \mathrm{N}_{2}$ at 2.0/1.2/1.5 $1 \mathrm{~min}^{-1}$. The mean power was $2.5 \mathrm{~W}$. Plasma treatment was carried out in a meander-like scanning mode with a line speed of 11,16 or $30 \mathrm{~mm} \mathrm{~s}^{-1}$ and a step width of $0.1 \mathrm{~mm}$. The temperature of the plasma jet on the surface of agar plates as well as on dentin slices was measured by means of infrared-camera thermoscopy (Optris PI; Optris). Temperature measurements were performed at room temperature with a thermal resolution of $\pm 0.1{ }^{\circ} \mathrm{C}$ at an optical frame of $160 \times 120$ pixels.

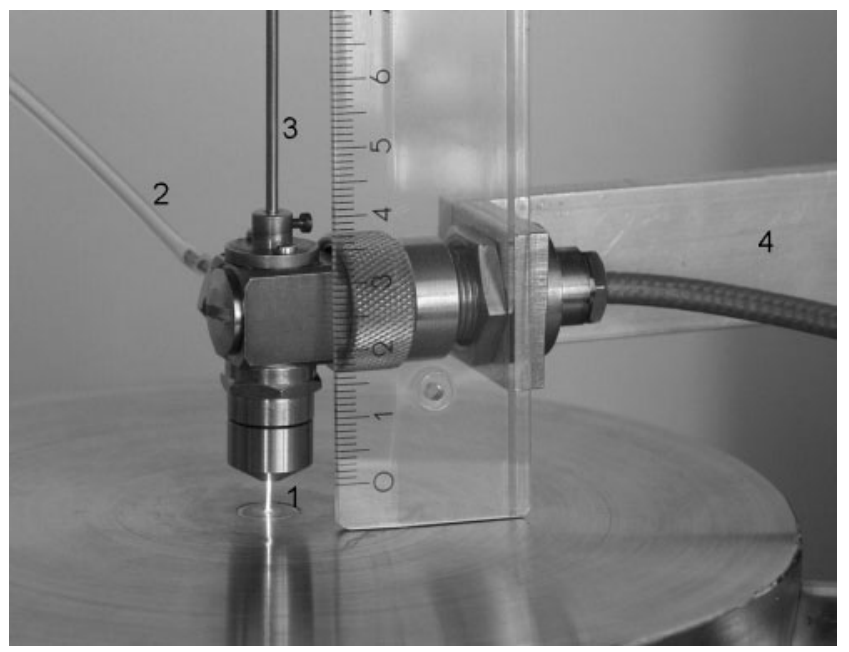

Fig. 1. Plasma source and plasma jet. 1, Plasma jet; 2 and 3 , the gas supply of the plasma source consists of an outer (2) and an inner gas inlet (3); 4 , the microwave to the plasma source.
Micro-organisms. The micro-organisms E. coli (XL1-Blue; Stratagene), L. casei (DSM 20011), S. mutans (DSM 20523) and C. albicans (DSM 1386) were cultivated overnight in liquid media ( $E$. coli and C. albicans, in LB broth, L. casei, in MRS broth, S. mutans, in brain heart infusion (BHI) broth) (all from Sigma-Aldrich). Their concentration was determined by measurement of the $\mathrm{OD}_{600}$ and revealed $8.7 \log _{10}$ c.f.u. $\mathrm{ml}^{-1}$ for E. coli, $8.7 \log _{10}$ c.f.u. $\mathrm{ml}^{-1}$ for $L$. casei and $8.5 \log _{10}$ c.f.u. $\mathrm{ml}^{-1}$ for C. albicans. The concentration of $S$. mutans was determined by plating tenfold dilution series on BHI agar and revealed $8.7 \log _{10}$ c.f.u. $\mathrm{ml}^{-1}$.

Agar plates and dentin slices. One-hundred microlitres of the four microbial suspensions were plated on ( $90 \mathrm{~mm}$ diameter) agar plates [E. coli on LB agar, L. casei on Rogosa agar, S. mutans on BHI agar (all from Sigma-Aldrich) and C. albicans on Sabouraud $4 \%$ glucose agar (from Carl Roth)] and dried for $15 \mathrm{~min}$ at $37^{\circ} \mathrm{C}$. A density of approximately $6 \log _{10}$ c.f.u. $\mathrm{cm}^{-2}$ resulted on the agar plates. The duration of the treatment of the agar plates was 33, 63 and $91 \mathrm{~s}$ in a meander-like scan of a surface area of $1 \mathrm{~cm}^{2}$ resulting in irradiation times of $0.3,0.6$ and $0.9 \mathrm{~s}$ per $\mathrm{mm}^{2}$, respectively. Agar plates were incubated for $48 \mathrm{~h}$ at $37^{\circ} \mathrm{C}$. The irradiated area was assessed in comparison to the untreated surrounding agar surface. Colonies were counted and inhibition zones were measured.

Dentin slices of $2 \mathrm{~mm}$ thickness with diameters of 8 to $10 \mathrm{~mm}$ were cut from the crowns of extracted human molars using a diamond rotating saw (Conrad Apparatebau). The exposed dentin was subjected to machine polishing (Metkon Gripo 2V; Metkon Instruments) with silicon-carbide grinding paper (Buehler) with decreasing grit size (grit P 120, 600, 800, 1200, 2500). Subsequently, the samples were cleaned ultrasonically for $15 \mathrm{~min}$, autoclaved at $121{ }^{\circ} \mathrm{C}$ and stored in $0.9 \% \mathrm{NaCl}$. Five microlitres of the four microbial suspensions were spotted on the centre of the dentin slices covering an area of approximately $20 \mathrm{~mm}^{2}$. The bacteria were allowed to adhere in a humid atmosphere for $15 \mathrm{~min}$ until plasma treatment was carried out. Dentin slices were fixed on sterile glass plates by wax in a marked area of $10 \times 10 \mathrm{~mm}$ and irradiated immediately. The area of $1 \mathrm{~cm}^{2}$ was treated for 33,63 and $91 \mathrm{~s}$ resulting in treatment times of $6 \mathrm{~s}\left(0.3 \mathrm{~s} \mathrm{~mm}^{-2}\right), 12 \mathrm{~s}\left(0.6 \mathrm{~s} \mathrm{~mm}^{-2}\right)$ and $18 \mathrm{~s}\left(0.9 \mathrm{~s} \mathrm{~mm}^{-2}\right)$ for the contaminated areas on the dentin slices, similar to the above described agar plate treatment. After plasma treatment dentin slices were vortexed for $1 \mathrm{~min}$ in $1 \mathrm{ml}$ liquid media, and $100 \mu \mathrm{l}$ of each of these samples were placed on agar plates in 10 -fold dilution series. The agar plates were incubated for $48 \mathrm{~h}$ at $37{ }^{\circ} \mathrm{C}$. After incubation, colonies on the agar plates were counted and the numbers of c.f.u. recovered from the dentin samples were calculated. The remaining $900 \mu \mathrm{l}$ liquid medium containing one dentin slice was incubated as described above in order to assess the disinfective efficacy of the plasma treatment. Samples without microbial growth after $48 \mathrm{~h}$ of incubation were assumed to be completely disinfected.

Scanning electron microscopy (SEM). In order to visualize the destruction of the micro-organisms due to plasma-jet treatment dentin slices were contaminated with microbial suspensions and the contaminated areas were plasma treated as described above. After irradiation, the samples were fixed in glutaraldehyde $(2.5 \%$ in PBS; PAA Laboratories) for $2 \mathrm{~h}$ and rinsed 5 times for $10 \mathrm{~min}$ in PBS. Subsequently, the samples were dehydrated in an increasing series of ethanol (50-90\% $10 \mathrm{~min}$ each; $100 \% 2 \times 10 \mathrm{~min}$ ). Finally, the samples were dried in 1,1,1,3,3,3-hexamethyl-disilazane (HMDS: Acros Organics). HMDS was vaporized at room temperature in a flowhood. Samples were mounted on SEM-sample stubs (Plano) and sputtered with gold/palladium $(20 \mathrm{~nm}$, Edwards Sputter Coater S 150B; Edwards). SEM assessment of the micro-organisms was performed with an ULTRA 55 scanning electron microscope (Carl Zeiss SMT). The dentin surfaces were scanned for bacterial remnants or intact bacteria. 
Control samples. Negative control samples (agar plates and dentin slices) were irradiated with the pure process gases without plasma ignition for $0.9 \mathrm{~s} \mathrm{~mm}^{-2}$. Additionally, areas of $1 \mathrm{~cm}^{2}$ on agar plates were irradiated with the plasma jet for $0.9 \mathrm{~s} \mathrm{~mm}^{-2}$ followed by subsequent plating of the four microbial suspensions in order to detect possible changes of the agar that could result in microbial growth inhibition. In addition, dentin slices were contaminated with the four micro-organisms. Micro-organisms not subjected to plasma treatment were recovered from the dentin slices as described above for control purposes.

Agar-plated micro-organisms were incubated with chlorhexidine (CHX) solution $(0.2 \%)$ for $90 \mathrm{~s}$ as positive controls. The CHX was removed, and the surfaces washed three times with liquid media. Agar plates were incubated as described above and the number of c.f.u. was determined. Contaminated dentin slices were incubated with $10 \mu \mathrm{l}$ CHX for $18 \mathrm{~s}$, corresponding to $0.9 \mathrm{~s} \mathrm{~mm}^{-2}$ plasma treatment. The CHX was removed; the surface of the dentin slices was carefully rinsed with PBS and the dentin slices were further processed as described above.

For SEM, untreated dentin slices and those irradiated without plasma ignition $\left(0.9 \mathrm{~s} \mathrm{~mm}^{-2}\right)$ were prepared as well.

Statistical analysis. Experiments were carried out five times, and the controls three times. All c.f.u. numbers were expressed as $\log _{10}$ values. The mean of the $\log _{10}$ values was computed together with $95 \%$ confidence intervals (CIs).

\section{RESULTS AND DISCUSSION}

\section{Temperature monitoring}

During irradiation of the dentin slice by the plasma jet, the dentin surface temperature increased instantaneously and reached its maximum in the jet's centre within $5 \mathrm{~s}$. A maximum temperature of $50.8^{\circ} \mathrm{C}$ was measured on the dentin surface without movement of the plasma jet over the sample. No further increase in temperature was observed. The temperature decreased to room temperature at a distance of $5 \mathrm{~mm}$ around the centre of the jet. Movement of the plasma jet resulted in a decrease of the maximum surface temperature in the centre of the plasma jet to values of 39 to $44{ }^{\circ} \mathrm{C}$. Lower temperatures were measured with increasing scan velocities. On the agar surface, the temperature of the plasma jet was $20{ }^{\circ} \mathrm{C}$ when unmoving and 14 to $20{ }^{\circ} \mathrm{C}$ under movement (Table 1).

Table 1. Temperatures measured on the surface of a dentin slice or agar plate without movement of the plasma jet, as well as under motion, with treatment times of $0.3,0.6$ or $0.9 \mathrm{~s} \mathrm{~mm}^{-2}$

Values are the mean and SD of tenfold measurements.

\begin{tabular}{|lcccc|}
\hline \multirow{2}{*}{$\begin{array}{l}\text { Surface } \\
\text { temp. }\left({ }^{\circ} \mathbf{C}\right)\end{array}$} & $\begin{array}{c}\text { No } \\
\text { movement }\end{array}$ & \multicolumn{3}{c|}{ Movement $\left(\mathbf{m m ~ s}^{-\mathbf{1}}\right)$} \\
\cline { 3 - 5 } & & $\mathbf{1 1}$ & $\mathbf{1 6}$ & $\mathbf{3 0}$ \\
\hline Dentin slice & $50.1 \pm 0.6$ & $43.6 \pm 1.2$ & $43.6 \pm 1.3$ & $39.1 \pm 1.0$ \\
Agar plate & $20.4 \pm 1.1$ & $19.5 \pm 0.8$ & $14.2 \pm 0.7$ & $14.8 \pm 0.5$ \\
\hline
\end{tabular}

\section{Plasma treatment of agar plates and dentin slices}

The plasma treatment of the agar plates caused complete growth inhibition of E. coli and L. casei on the irradiated surface areas. Between one and four colonies were present on $30 \%$ of the treated areas on agar plates inoculated with C. albicans. A time-dependent reduction of growth was observed on the irradiated surfaces for $S$. mutans. A reduction of colony density was observed after irradiation at $0.3 \mathrm{~s} \mathrm{~mm}^{-2}$ in comparison to untreated control areas. A mean of $1.3 \log _{10}$ c.f.u. was present after $0.6 \mathrm{~s} \mathrm{~mm}^{-2}$, whereas the c.f.u. number dropped to a mean lower than 1 $\log _{10}$ after $0.9 \mathrm{~s} \mathrm{~mm}^{-2}$ of irradiation (Fig. 2). Expanded inhibition zones surrounding the irradiated areas were observed. These inhibition zones were most distinctive for L. casei, whereas agar plates infected with C. albicans and E. coli demonstrated less widespread inhibition zones. Inhibition zones were smallest for $S$. mutans. The extent of the surrounding inhibition zones was correlated with the length of irradiation. Longer irradiation times resulted in extended inhibition zones (Table 2).

CHX treatment resulted in complete microbial growth inhibition on the agar. No inhibition of microbial growth was visible on the agar plates irradiated with only the process gases. Plasma treatment of the agar plates before microbial inoculation did not influence microbial growth (Table 2).

A mean of $2 \log _{10}$ c.f.u. of E. coli and L. casei were recovered from dentin slices after plasma treatment for $0.3 \mathrm{~s} \mathrm{~mm}^{-2}$. After 0.6 and $0.9 \mathrm{~s} \mathrm{~mm}^{-2}$ of treatment this number decreased to $1 \log _{10}$ c.f.u. Independently of the irradiation time, a mean of $1 \log _{10}$ c.f.u. was isolated for $C$. albicans. In contrast, a c.f.u. reduction dependent on the duration of irradiation was observed for S. mutans. Their mean numbers decreased from $3 \log _{10}$ c.f.u. after $0.3 \mathrm{~s}$

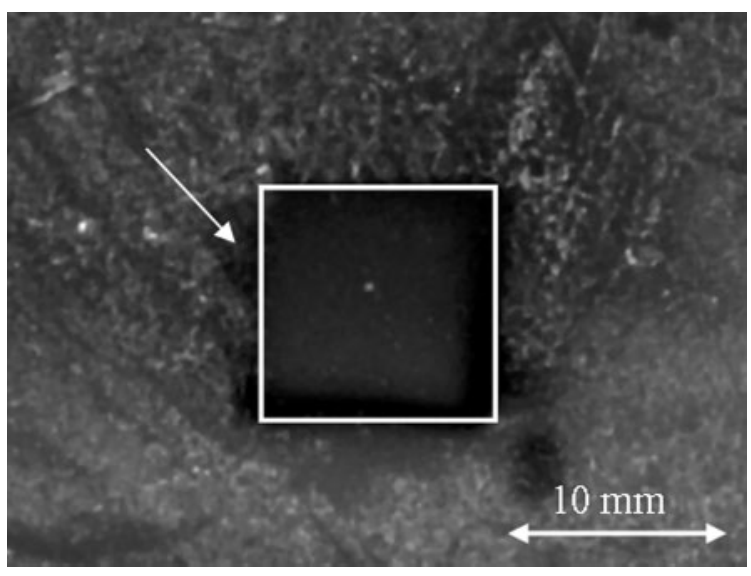

Fig. 2. Plasma treated $\left(0.9 \mathrm{~s} \mathrm{~mm}^{-2}\right)$ area of $1 \mathrm{~cm}^{2}$ (indicated by a white box) on an agar plate inoculated with S. mutans after $48 \mathrm{~h}$ incubation at $37{ }^{\circ} \mathrm{C}$. The colony number in the treated area was 1 $\log _{10}$ c.f.u. Also an expanded growth inhibition zone of 1 to $2 \mathrm{~mm}$ surrounding the irradiation area is visible (indicated by an arrow). 
Table 2. Effect of the plasma treatment $(P)$ on agar plates inoculated with $100 \mu \mathrm{l}$ of $8.7 \log _{10}$ c.f.u. of the micro-organisms $E$. coli, $L$. casei, S. mutans and C. albicans, and results of $\mathrm{CHX}$ application, plasma treatment of agar before microbial plating (PB) and application of process gases without plasma ignition $(\mathrm{G})$

All treatments were carried out with treatment times of $0.3,0.6$ or $0.9 \mathrm{~s} \mathrm{~mm}{ }^{-2}$ on agar areas of $1 \mathrm{~cm}^{2}$, and all agar plates were incubated at $37{ }^{\circ} \mathrm{C}$ for $48 \mathrm{~h}$. The mean number of colonies (mean $\log _{10}$ c.f.u.) in the treated areas $(n \pm 95 \% \mathrm{CI})$ and mean expanded inhibition zones (expIZ) around the treated areas $(\mathrm{mm} \pm \mathrm{SD})$ are given. NGI, No growth inhibition visible; NC, no colonies detectable.

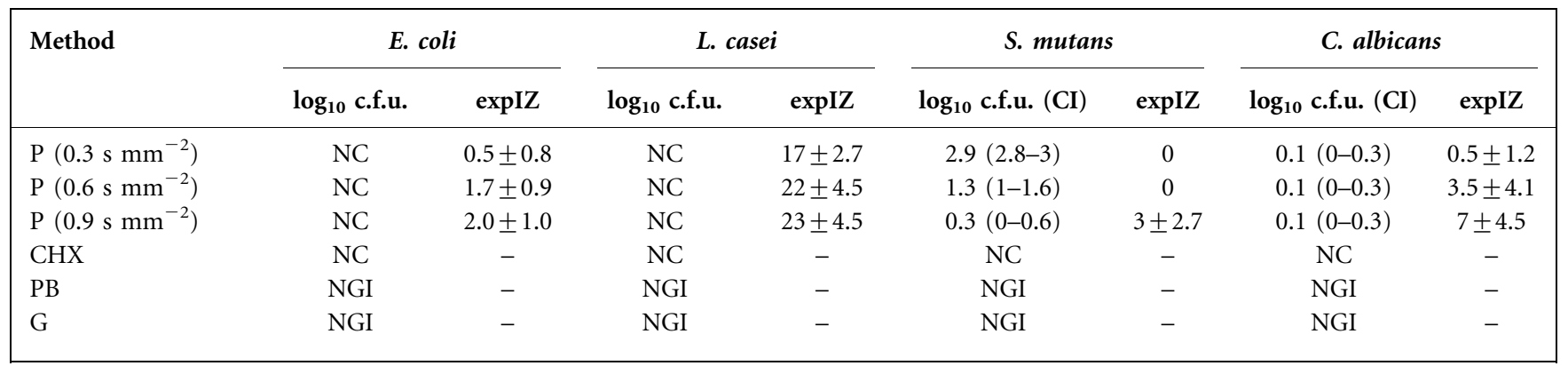

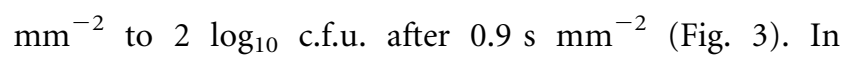
correlation to longer plasma-jet irradiation times increasing numbers of dentin slices were completely disinfected, which was assessed by incubation of the dentin slices in liquid medium for $48 \mathrm{~h}$. The plasma jet was most effective against $C$. albicans and E. coli after irradiation of 0.6 and $0.9 \mathrm{~s} \mathrm{~mm}^{-2}$. No microbial growth was observed in up to four of five liquid samples. Only one of five dentin slices inoculated with S. mutans were found to be completely disinfected. CHX treatment resulted in comparable reductions of microbes on dentin slices. No c.f.u. of C. albicans were isolated from the dentin slices and the according liquid cultures were free of microbial growth. In the samples infected with L. casei $1 \log _{10}$ c.f.u. were found, for E. coli between 1 and $2 \log _{10}$ c.f.u. were found and for $S$. mutans $2 \log _{10}$ c.f.u. were detected in one or two not
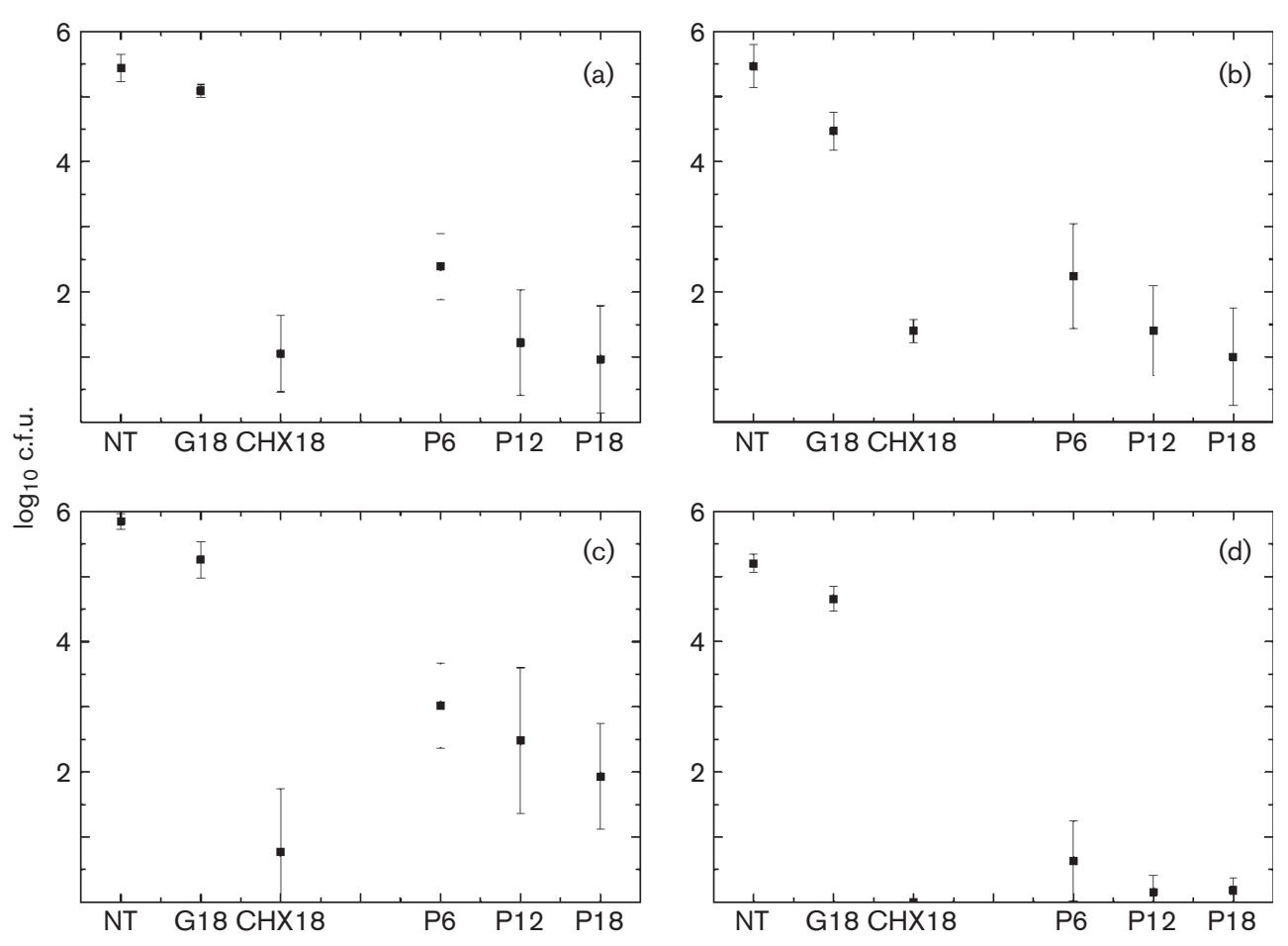

Fig. 3. Killing of adherent micro-organisms on dentin slices with a cold atmospheric plasma jet. The c.f.u. values are given in $\log _{10}$ c.f.u. of E. coli (a), L. casei (b), S. mutans (c) and C. albicans (d) recovered after plasma treatment. P6, P12, P18, indicate exposure to the plasma jet for 6,12 or $18 \mathrm{~s}\left(0.3,0.6\right.$ or $0.9 \mathrm{~s} \mathrm{~mm}^{-2}$, respectively); G18, gas irradiation without plasma ignition for $18 \mathrm{~s}\left(0.9 \mathrm{~s} \mathrm{~mm}^{-2}\right) ; \mathrm{CHX} 18$, exposure to $\mathrm{CHX}$ for $18 \mathrm{~s}$; NT, no treatment. Mean values with $95 \%$ Cls are the mean of a fivefold determination for plasma-jet-treated samples and of triplicates for the controls. 
completely disinfected dentin slices each. Between 4 and 5 $\log _{10}$ c.f.u. were recovered from the samples irradiated only with the process gases, and more than $5 \log _{10}$ c.f.u. were isolated from the untreated controls. All liquid samples showed distinct microbial growth (Table 3, Fig. 3).

\section{SEM analysis}

Plasma treatment induced distinct micromorphological alterations of adherent micro-organisms. Only microbial remnants, cells with pores in their cell walls and a low number of intact micro-organisms were detected on the dentin surfaces after plasma-jet treatment independently of the application time (Fig. 4a, b, c, d). However, no complete removal of adherent micro-organisms from the dentin surfaces was observed. In contrast, intact microorganisms dominated on dentin samples treated without plasma ignition or on untreated dentin surfaces, and no reduction of the number of adherent micro-organisms could be observed.

The present investigation demonstrated that a cold atmospheric plasma jet is suitable for substantially reducing oral micro-organisms on agar plates or adherent to dentin slices, as well as the bacterium E. coli. These results confirm previously published data on the efficacy of cold plasma jets for killing and removal of planktonic or adherent micro-organisms (Lee et al., 2006; Sladek et al., 2007; Hong et al., 2009). Parts of agar plates contaminated with a density of $6 \log _{10}$ c.f.u. $\mathrm{cm}^{-2}$ were nearly completely disinfected by plasma-jet treatment in the present study. Disinfection on directly irradiated areas was achieved at the shortest treatment time for E. coli, L. casei and C. albicans. For S. mutans, however, longer treatment times were necessary. Surrounding the directly irradiated areas of $1 \mathrm{~cm}^{2}$, additional, irregular inhibition zones were recorded

Table 3. Number of completely disinfected dentin slices inoculated with $6.4 \log _{10}$ c.f.u. after subsequent plasma-jet treatment $(P)$ (exposure to plasma jet at $0.3,0.6,0.9 \mathrm{~s} \mathrm{~mm}^{-2}$ ), $\mathrm{CHX}$ treatment for $18 \mathrm{~s}(\mathrm{CHX})$, application of process gases without plasma ignition for $18 \mathrm{~s}(\mathrm{G})$ or no treatment (NT)

Liquid medium containing dentin slices ( $n=5$ for plasma treatment, $n=3$ for controls) was incubated for $48 \mathrm{~h}$ at $37^{\circ} \mathrm{C}$ to detect bacterial growth.

\begin{tabular}{|ccccc|}
\hline Method & E. coli & L. casei & S. mutans & C. albicans \\
\hline $\begin{array}{c}\text { P (0.3 s } \\
\left.\mathrm{mm}^{-2}\right)\end{array}$ & $0 / 5$ & $1 / 5$ & $0 / 5$ & $2 / 5$ \\
$\mathrm{P}(0.6 \mathrm{~s}$ & $2 / 5$ & $1 / 5$ & $1 / 5$ & $4 / 5$ \\
$\left.\mathrm{~mm}^{-2}\right)$ & & & & \\
P (0.9 s & $3 / 5$ & $2 / 5$ & $1 / 5$ & $3 / 5$ \\
$\left.\mathrm{~mm}^{-2}\right)$ & & & & \\
$\mathrm{CHX}(18 \mathrm{~s})$ & $2 / 3$ & $1 / 3$ & $2 / 3$ & $3 / 3$ \\
$\mathrm{G}(18 \mathrm{~s})$ & $0 / 3$ & $0 / 3$ & $0 / 3$ & $0 / 3$ \\
$\mathrm{NT}$ & $0 / 3$ & $0 / 3$ & $0 / 3$ & $0 / 3$ \\
\hline
\end{tabular}

for all the micro-organisms, which expanded after longer plasma treatment. The appearance of inhibition zones outside of the directly irradiated area suggests an antimicrobial effect of the plasma jet beyond the directly treated areas. This extension of the inhibition zones might be caused by reactive oxygen species produced by the plasma jet (Hong et al., 2009).

A substantial reduction of approximately $4 \log _{10}$ intervals was observed on the dentin slices for the bacteria E. coli, $L$. casei and $S$. mutans, and even slightly higher killing rates for C. albicans, after plasma-jet treatment. The destructive power of the plasma jet against the four micro-organisms tested in the present study on dentin slices was visualized by SEM micrographs. On some of the dentin slabs, the adherent micro-organisms were completely destroyed. However, killing of the microbes by plasma jet on the dentin slices was not as effective as treatment of agar plates. This might be caused by the penetration of the microorganisms into the dentin tubules before plasma treatment during sample drying. Additionally, micro-organisms located at the dentin tubules orifices might be transported into the inner dentin by the gas pressure of the plasma jet.

Distinct differences in the susceptibilities of the four investigated micro-organisms to cold plasma irradiation were detected in the present study. S. mutans revealed the strongest resistance to plasma-jet irradiation on agar plates, as well as on dentin slices. S. mutans is a Gram-positive, non-motile facultative anaerobe and small in size compared with the other investigated micro-organisms. Its relative resistance to plasma-jet treatment may be explained by its small size. Presumably it was transported into the dentin tubules due to the gas pressure of the plasma jet. Another explanation could be the structure of the cell wall of $S$. mutans, which is composed of highly cross-linked murein. The particular influence of reactive oxygen species or of the temperature of the plasma jet cannot be elucidated from this study. The killing rate on the dentin slices increased during irradiation of $0.9 \mathrm{~s}$ $\mathrm{mm}^{-2}$ with a related temperature of $44^{\circ} \mathrm{C}$, compared to an irradiation time of $0.3 \mathrm{~s} \mathrm{~mm}^{-2}$ with maximum temperature lower than $40{ }^{\circ} \mathrm{C}$. The same correlation was observed for microbes on agar plates combined with expanding inhibition zones at longer irradiation times. In conclusion of these results, direct and indirect co-effects of the plasma jet caused its anti-microbial efficacy.

Undoubtedly, additional disinfecting effects up to a complete sterilization of surfaces may be achieved by a plasma jet at higher performances correlated with higher temperatures and extended treatment times. However, there are certain limitations concerning these parameters for the application of the plasma-jet technology under oral conditions, and especially when dentin is treated. The combination of gas stream, chemical radicals and electrons can cause severe desiccation of dentin resulting in cracks and damage of odontoblasts or pulp tissue. For these reasons, we chose experimental conditions simulating 
(a)

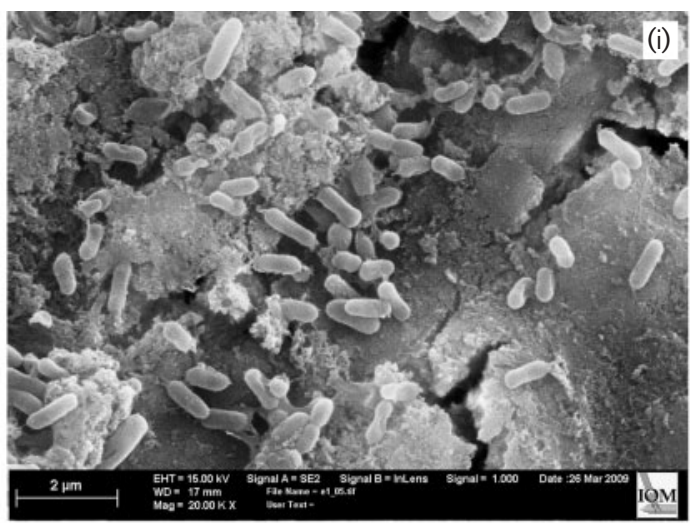

(b)

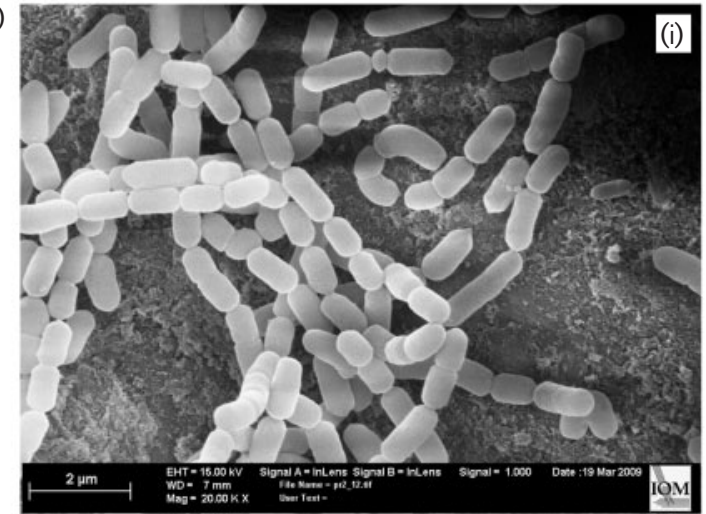

(c)

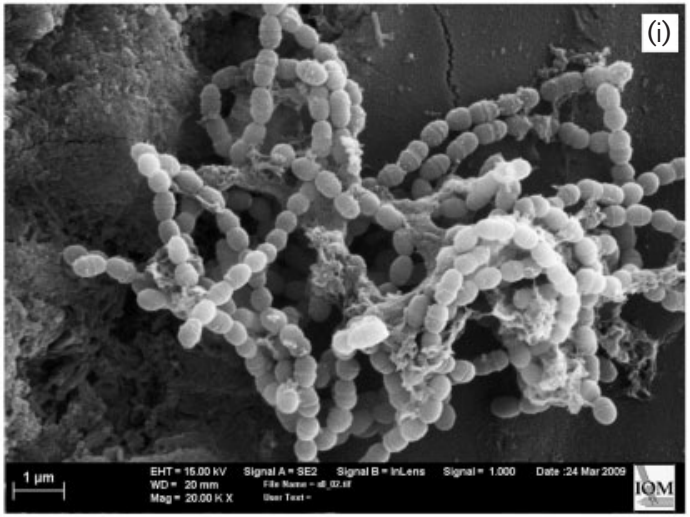

(d)

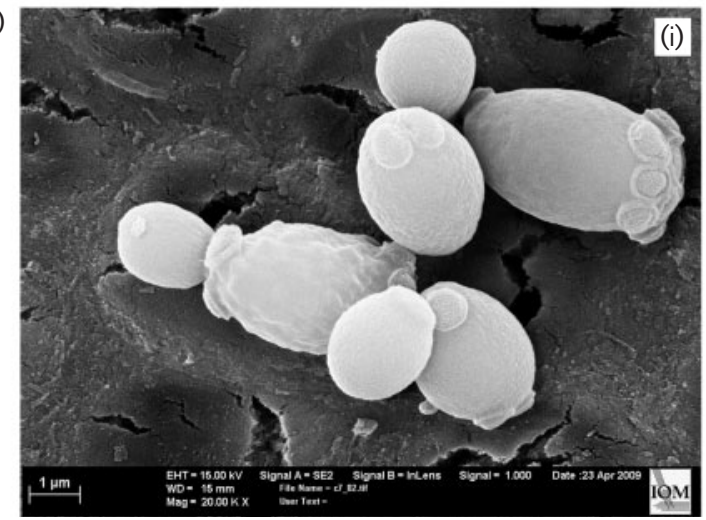

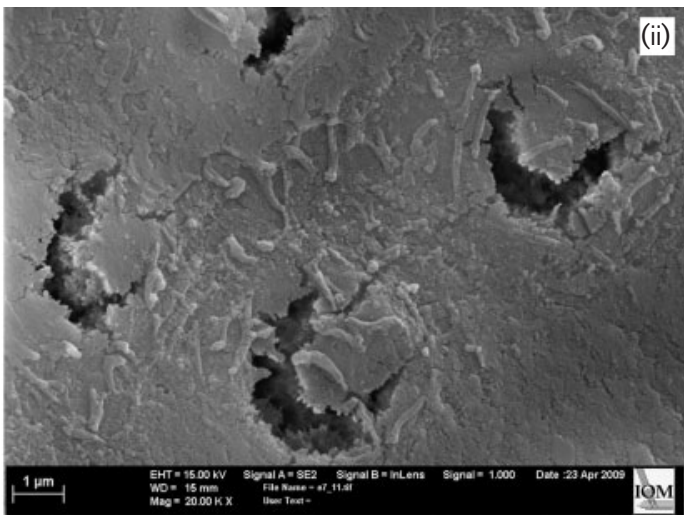
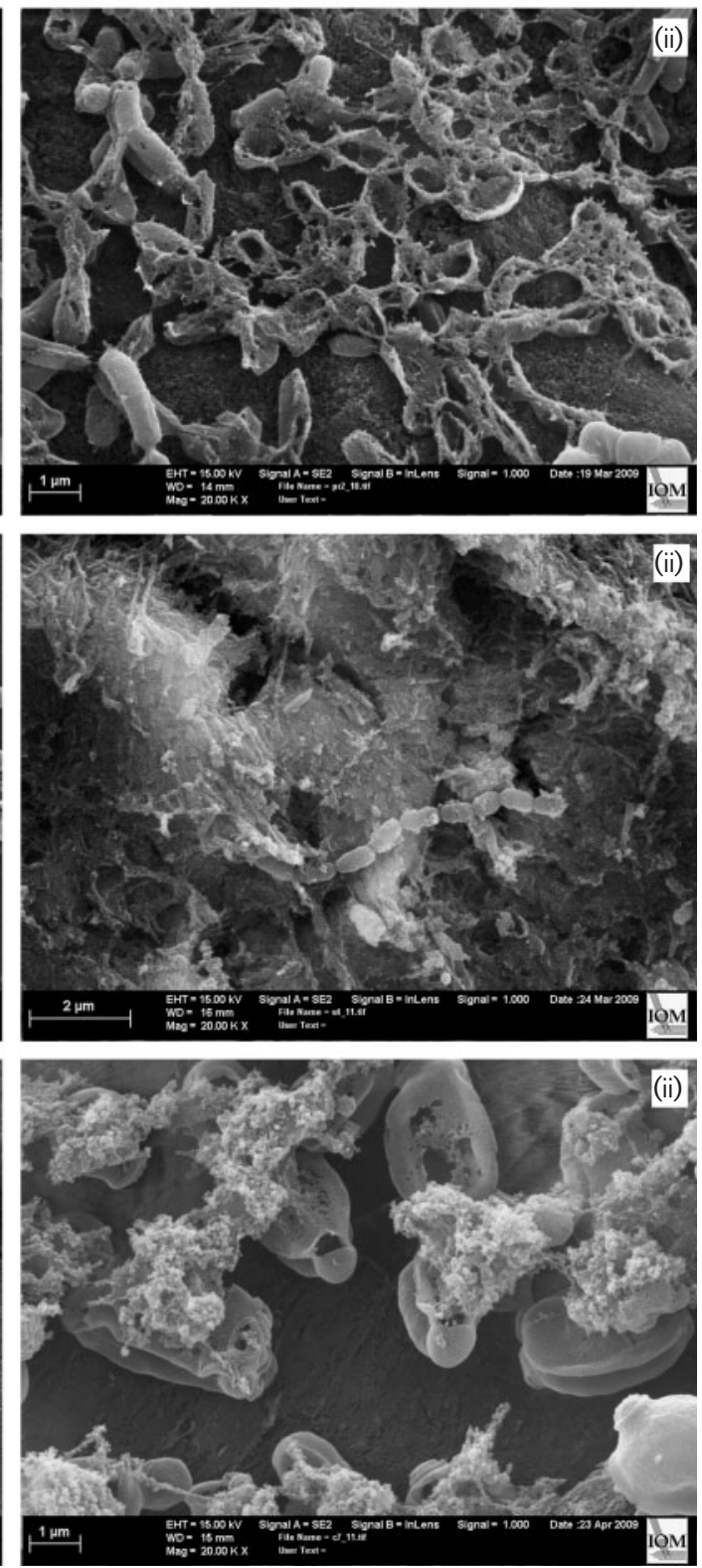

Fig. 4. Representative scanning electron micrographs of adherent micro-organisms on dentin slices: (i) controls without plasma treatment and (ii) after plasma treatment $\left(0.9 \mathrm{~s} \mathrm{~mm}^{-2}\right.$ ). (a) E. coli, (b) L. casei, (c) S. mutans and (d) C. albicans. Magnification $\times 20000$. 
realistic oral dental conditions to prevent heat-induced damage to the dentin and pulp. The plasma jet was applied under meander-like motion to prevent temperature increase and dehydration of the dentin. Application times of $5 \mathrm{~s}$ are common in dentistry, e. g. for air drying of cavities. Temperature rises of $5{ }^{\circ} \mathrm{C}$ in the pulp chamber have been reported for the use of light-curing units currently used in restorative dentistry (Hannig \& Bott, 1999; Miletic et al., 2009). Concerning these aspects, the plasma jet used in the present study appeared to be applicable to dental surfaces. An improvement of the plasma jet might be achieved by further optimization of the mixture of noble and reactive gases, as well as flow conditions by modification of the plasma source. These aspects are currently under investigation.

Despite all limitations shown in the present study, plasmajet applications are of great interest for dentistry. The technology offers the benefits that were expected from ozone treatment but that so far have not been proven (Baysan \& Beighton, 2007). The results of our investigation emphasize the potential of cold plasma jets for antimicrobial applications in dentistry. In particular, the disinfection of infected dentin after caries removal or of shallow root caries lesions by plasma-jet treatment might be an interesting option.

\section{ACKNOWLEDGEMENTS}

This study was supported by grants from the Federal Ministry of Education and Research Germany (BMBF; FKZ 01 EZ 0730 and 01 EZ 0731). We thank Mrs Tina Hofmann, Mr Dietmar Hirsch, Ms Kathrin Schmidt and Ms Silke Gädt for technical assistance, as well as Ms Anja Niemeyer-Rupf and Ms Sonya Faber for critical reading of the manuscript.

\section{REFERENCES}

Badet, C. \& Thebaud, N. B. (2008). Ecology of lactobacilli in the oral cavity: a review of literature. Open Microbiol J 2, 38-48.

Baysan, A. \& Beighton, D. (2007). Assessment of the ozone-mediated killing of bacteria in infected dentine associated with non-cavitated occlusal carious lesions. Caries Res 41, 337-341.

Becker, K., Koutsospyros, A., Yin, S.-M., Christodoulatos, C., Abramzon, N., Joaquin, J. C. \& Brelles-Mariño, M. (2005). Environmental and biological applications of microplasmas. Plasma Phys Contr Fusion 47, B513-B523.

Coulombe, S., Léveillé, V., Yonson, S. \& Leask, R. L. (2006). Miniature atmospheric pressure glow discharge torch (APGD- $t$ ) for local biomedical applications. Pure Appl Chem 78, 1147-1156.

Duan, Y., Huang, C. \& Yu, Q. S. (2007). Cold plasma brush generated at atmospheric pressure. Rev Sci Instrum 78, 015104.

Goree, J., Liu, B., Drake, D. \& Stoffels, E. (2006). Killing of S. mutans bacteria using a plasma needle at atmospheric pressure. IEEE Trans Plasma Sci 34, 1317-1324.

Hannig, M. \& Bott, B. (1999). In-vitro pulp chamber temperature rise during composite resin polymerization with various light-curing sources. Dent Mater 15, 275-281.

Hong, Y. F., Kang, J. G., Lee, H. Y., Uhm, H. S., Moon, E. \& Park, Y. H. (2009). Sterilization effect of atmospheric plasma on Escherichia coli and Bacillus subtilis endospores. Lett Appl Microbiol 48, 33-37.
Kieft, I. E., van Berkel, J. J. B. N., Kieft, E. R. \& Stoffels, E. (2005). Radicals of plasma needle detected with fluorescent probe. In Plasma Processes and Polymers, chapter 22, pp. 295-308. Edited by R. d'Agostino, P. Favia, C. Oehr \& M. R. Wertheimer. Berlin: Wiley-VCH. Kim, S. M. \& Kim, J. I. (2006). Decomposition of biological macromolecules by plasma generated with helium and oxygen. J Microbiol 44, 466-471.

Klinke, T., Kneist, S., de Soet, J. J., Kuhlisch, E., Mauersberger, S., Forster, A. \& Klimm, W. (2009). Acid production by oral strains of Candida albicans and lactobacilli. Caries Res 43, 83-91.

Laroussi, M. (2002). Nonthermal decontamination of biological media by atmospheric-pressure plasmas: review, analysis, and prospects. IEEE Trans Plasma Sci 30, 1409-1415.

Laroussi, M. \& Leipold, F. (2004). Evaluation of the roles of reactive species, heat, and UV radiation in the inactivation of bacterial cells by air plasmas at atmospheric pressure. Int J Mass Spectrom 233, 81-86.

Laroussi, M. \& Lu, X. (2005). Room-temperature atmospheric pressure plasma plume for biomedical applications. Appl Phys Lett 87, 113902.

Lee, K., Paek, K. H., Ju, W. T. \& Lee, Y. (2006). Sterilization of bacteria, yeast, and bacterial endospores by atmospheric-pressure cold plasma using helium and oxygen. J Microbiol 44, 269-275.

Manner, H. (2008). Argon plasma coagulation therapy. Curr Opin Gastroenterol 24, 612-616.

Masaoka, S. (2007). Plasma sterilization of polyethylene terephthalate bottles by pulsed corona discharge at atmospheric pressure. Biocontrol Sci 12, 59-63.

Miletic, V., Ivanovic, V., Dzeletovic, B. \& Lezaja, M. (2009). Temperature changes in silorane-, ormocer-, and dimethacrylatebased composites and pulp chamber roof during light-curing. J Esthet Restor Dent 21, 122-132.

Moisan, M., Barbeau, J., Moreau, S., Pelletier, J., Tabrizian, M. \& Yahia, L. H. (2001). Low-temperature sterilization using gas plasmas: a review of the experiments and an analysis of the inactivation mechanisms. Int J Pharm 226, 1-21.

Rupf, S., Hannig, M., Breitung, K., Schellenberger, W., Eschrich, K., Remmerbach, T. \& Kneist, S. (2008). Phenotypic heterogeneity of Streptococcus mutans in dentin. J Dent Res 87, 1172-1176.

Sladek, R. E. J., Stoffels, E., Walraven, R., Tielbeek, P. J. A. \& Koolhoven, R. A. (2004). Plasma treatment of dental cavities: a feasibility study. IEEE Trans Plasma Sci 32, 1540-1543.

Sladek, R. E., Filoche, S. K., Sissons, C. H. \& Stoffels, E. (2007). Treatment of Streptococcus mutans biofilms with a nonthermal atmospheric plasma. Lett Appl Microbiol 45, 318-323.

Stoffels, E., Flikweert, A. J., Stoffels, W. W. \& Kroesen, G. M. W. (2002). Plasma needle: a non-destructive atmospheric plasma source for fine surface treatment of (bio)materials. Plasma Sources Sci Technol 11, 383-388.

Venezia, R. A., Orrico, M., Houston, E., Yin, S. M. \& Naumova, Y. Y. (2008). Lethal activity of nonthermal plasma sterilization against microorganisms. Infect Control Hosp Epidemiol 29, 430-436.

Vleugels, M., Shama, G., Deng, X. T., Greenacre, E., Brocklehurst, T. \& Kong, M. G. (2005). Atmospheric plasma inactivation of biofilm-forming bacteria for food safety control. IEEE Trans Plasma Sci 33, 824-828.

Yu, H., Perni, S., Shi, J. J., Wang, D. Z., Kong, M. G. \& Shama, G. (2006). Effects of cell surface loading and phase of growth in cold atmospheric gas plasma inactivation of Escherichia coli K12. J Appl Microbiol 101, 1323-1330.

Yu, Q. S., Huang, C., Hsieh, F. H., Huff, H. \& Duan, Y. (2007). Bacterial inactivation using a low-temperature atmospheric plasma brush sustained with argon gas. J Biomed Mater Res B Appl Biomater 80, 211-219. 